\title{
An environmental scan of quality improvement and patient safety activities in emergency medicine in Canada
}

\author{
Edmund S.H. Kwok, MD, MHA, MSc ${ }^{*}$; Jeffrey J. Perry, MD, MSc ${ }^{*}$; Shawn Mondoux, MD, MSc ${ }^{\ddagger \S}$; \\ Lucas B. Chartier, MDCM, MPH»॥
}

\begin{abstract}
CLINICIAN'S CAPSULE
What is known about the topic?

Despite growing interest in quality improvement and patient safety (QIPS), it is unclear how individual emergency departments (ED) are supporting these activities. What did this study ask?

What is the current state of the QIPS infrastructure and activities in academic emergency medicine departments across Canada?

What did this study find?

This survey of department chiefs and QIPS leads found significant educational and academic efforts, with inconsistent levels of formal support/infrastructure.

Why does this study matter to clinicians?

This study highlights potential opportunities to advance QIPS efforts in emergency medicine further.
\end{abstract}

\section{ABSTRACT}

Objective: We conducted an environmental scan of quality improvement and patient safety (QIPS) infrastructure and activities in academic emergency medicine (EM) programs and departments across Canada.

Methods: We developed 2 electronic surveys through expert panel consensus to assess important themes identified by the CAEP QIPS Committee. "Survey 1" was sent by email to all 17 Canadian medical school affiliated EM department Chairs and Academic Hospitals department Chiefs; "Survey 2" to 12 identified OIPS leads in these hospitals. This was followed by 2 monthly email reminders to participate in the survey.

Results: 22/70 (31.4\%) Department Chairs/Chiefs completed Survey 1. Most (81.8\%) reported formal positions dedicated to QIPS activities within their groups, with a mixed funding model. Less than half of these positions have dedicated logistical support. $11 / 12(91.7 \%)$ local QIPS leads completed Survey 2. Two-thirds $(63.6 \%)$ reported explicit QIPS topics within residency curricula, but only $9.1 \%$ described QIPS training for staff physicians. Many described successful academic scholarship output, with the total number of peer-reviewed QIPS-related publications per centre ranging from 1-10 over the past 5 years. Few respondents reported access to academic supports: methodologists $(27.3 \%)$, administrative personnel $(27.3 \%)$, and statisticians (9.1\%).

Conclusion: This environmental scan provides a snapshot of OIPS activities in EM across academic centres in Canada. We found significant local educational and academic efforts, although there is a discrepancy between the level of formal support/infrastructure and such activities. There remains opportunity to further advance QIPS efforts on a national level, as well as advocating and supporting local QIPS activities.

\section{RÉSUMÉ}

Objectif: L'étude visait à réaliser une analyse environnementale des activités d'amélioration de la qualité de la pratique clinique et de la sécurité des patients (AQSP) ainsi que de l'infrastructure afférente dans les programmes de médecine d'urgence (MU) et dans les services des urgences des hôpitaux d'enseignement partout au Canada.

Méthode: Deux questionnaires d'enquête électroniques ont été élaborés par un groupe d'experts après l'atteinte d'un consensus dans le but d'évaluer des thèmes jugés importants par le comité de I'AOSP de I'Association canadienne des médecins d'urgence. Le premier questionnaire a été envoyé par courriel aux directeurs de département de $\mathrm{MU}$ et aux chefs de service des hôpitaux d'enseignement rattachés

From *Department of Emergency Medicine, University of Ottawa, Ottawa, ON; †Ottawa Hospital Research Institute, Ottawa, ON; $\neq D e p a r t m e n t ~ o f$ Emergency Medicine, Hamilton Health Science, Hamilton, ON; §Division of Emergency Medicine, Department of Medicine, McMaster University, Hamilton, ON; TDepartment of Emergency Medicine, University Health Network, Toronto, ON; and the \|Division of Emergency Medicine, Department of Medicine, University of Toronto, Toronto, ON.

Correspondence to: Dr. Edmund S.H. Kwok, Department of Emergency Medicine, The Ottawa Hospital, 1053 Carling Ave, Ottawa, ON, K1Y 4E9; Email: ekwok@toh.ca

(C) Canadian Association of Emergency Physicians 
aux 17 écoles de médecine au Canada; le deuxième, à 12 responsables des activités d'AQSP, désignés comme tels, dans ces hôpitaux. Ont suivi deux rappels envoyés par courriel, à un mois d'intervalle, aux participants concernés.

Résultats: Au total, 22 directeurs de département ou chefs de service sur $70(31,4 \%)$ ont répondu au premier questionnaire. La grande majorité d'entre eux $(81,8 \%)$ ont fait état de postes officiels réservés aux activités d'AQSP dans leur groupe, soutenus selon un modèle de financement mixte. Toutefois, moins de la moitié des postes en question disposent d'un soutien logistique particulier. Quant au deuxième questionnaire, 11 responsables locaux des activités d'AOSP sur $12(91,7 \%)$ y ont répondu. Environ les deux tiers $(63,6 \%)$ ont indiqué que des sujets explicites d'AQSP étaient inclus dans les programmes de résidence, mais seulement $9,1 \%$ des responsables ont décrit la formation en matière d'AQSP donnée aux médecins membres du personnel hospitalier. Par ailleurs, bon nombre de répondants ont fait état de travaux d'érudition couronnés de succès; ainsi, le nombre total de publications évaluées par les pairs en lien avec I'AQSP variait de 1 à 10 par centre, au cours des 5 dernières années. Enfin, peu de répondants ont indiqué bénéficier du soutien de ressources universitaires : spécialistes de la méthodologie $(27,3 \%)$, personnel administratif $(27,3 \%)$ et statisticiens $(9,1 \%)$.

Conclusion: Cette analyse environnementale a dressé le portrait des activités d'AOSP menées dans les services de MU dans les hôpitaux d'enseignement au Canada. Les efforts fournis sur les plans de la formation et du soutien universitaire à l'échelle locale sont importants, mais il y a un déséquilibre entre le degré de soutien structuré ou d'infrastructure disponible et la réalisation de ces activités. Aussi y a-t-il lieu d'accroître les efforts d'AQSP à l'échelle nationale, en plus de soutenir ces activités à l'échelle locale et d'en faire la promotion.

Keywords: Emergency medicine, environmental scan, patient safety, quality improvement

\section{INTRODUCTION}

Quality improvement and patient safety (QIPS) activities in health care have become increasingly important in recent years, driven, in part, by a focus on preventable medical errors. ${ }^{1-5}$ Initially, the term "quality improvement" was loosely defined as "the combined efforts to make the changes that will lead to better patient outcomes, system performance, and professional development." Specifically, for this paper, QIPS refers to a range of activities utilizing methodological and statistical rigour through which health care providers develop, implement, and assess small-scale interventions, identify those that work well, and implement them more broadly to improve clinical practice and patient safety. ${ }^{7}$ Encouraged by various local, national, and international health care organizations, individual hospitals and medical groups have engaged in QIPS activities across Canada., 8 Previously published QIPS efforts within emergency medicine (EM) in Canada have targeted overcrowding, wait times, and resource utilization. ${ }^{10-16}$ The Canadian Fournal of Emergency Medicine (CFEM) has also recently published a three-paper series on QI, to enable EM practitioners to learn and apply relevant methods to improve care locally. ${ }^{17-19}$ However, it is unclear what the current national landscape is with regards to how individual EM departments are supporting QIPS activities and evaluating their success and sustainability.

The Canadian Association of Emergency Physicians (CAEP) recently formed a dedicated QIPS Committee tasked with providing leadership and advocacy with issues relating to quality and safety in EM across Canada. A panel for the 2018 Academic Symposium on Leadership was formed to examine the barriers, facilitators, and current national context for the pursuit of QIPS activities in EM. The results of this initiative were presented at the 2018 Symposium in Calgary and have been published concurrently with this article in $C 7 E M .^{20}$ One of the major gaps identified early on through this initiative was the lack of understanding of how EM departments at each major academic teaching medical centre engage in QIPS activities. Specifically, knowledge gaps abounded with respect to funding, infrastructure, personnel, education, and academic support. Having a better understanding of the strengths and existing gaps would help guide national leadership on developing recommendations to enhance QIPS progress and patient care further in EM across Canada.

We sought to assess how Canadian medical school EM departments/divisions and major Canadian teaching hospitals approach QIPS programs and efforts, with regards to training, available infrastructure, education, scholarly activities, and perceived needs.

\section{METHODS}

We conducted an electronic survey inviting all 17 Canadian university-affiliated EM department/division chairs and affiliated academic hospital emergency department (ED) chiefs, as well as locally identified EM QIPS leads. 


\section{Survey design}

Through a literature review and assessment of prior surveys of other academic EM activities in Canada, ${ }^{18,19}$ QIPS experts (EK, LBC, and SM) and a survey methodologist (JJP) generated survey questions around core themes deemed important by the CAEP QIPS committee. These themes included formal training and skill capacity, operational infrastructure, educational activities, academic and scholarship, and perceived gaps and needs.

These questions were then divided into two separate surveys targeted toward the specific individuals who would be most appropriately positioned to answer the respective questions. The surveys were comprised of quantitative and qualitative items, as well as comment boxes, and they were thoroughly reviewed and revised by the authors for clarity and flow logic. Survey 1 comprised 21 questions focusing on "formal training and skill capacity," "operational infrastructure," and "perceived gaps and needs," meant for department/division chairs and ED chiefs (see Appendix A). Survey 2 comprised 33 questions focusing on more front-line operations including "educational activities," "academic and scholarship," "QIPS activities," and "perceived gaps and needs," meant for local QIPS leads (see Appendix B).

\section{Recruitment}

We included all 17 Canadian universities with a medical school and their affiliated academic hospitals. We identified individual EM department/division academic chairs at each medical school and ED chiefs at each affiliated academic hospital, through their respective organization's website contact information, personal communication, or both. An initial email was sent in February 2018 to these individuals to invite them to participate in Survey 1 , followed by two separate reminder emails once a month. In addition, we asked each of these chairs/chiefs to further identify within their group any individual(s) currently responsible for QIPS activities at their site. A separate email invitation was then sent to these local QIPS leads, inviting them to participate in Survey 2. The methods for the second survey were the same, with a reminder email sent monthly for two additional months.

\section{Survey administration and data collection}

We administered the survey electronically using SurveyMonkey 2018 (SurveyMonkey Canada Inc., Ottawa,
$\mathrm{ON})$. A link to the survey was included in the recruitment emails sent to the participants. Participation was voluntary, and all responses were anonymous, with no identifying information linked to respondents. Results from the survey were electronically collected into a Microsoft Excel 2017 (Microsoft Corp., Redmond, WA) downloadable database for analysis. We present descriptive statistics including proportions, means, medians, and ranges, as appropriate. Qualitative comments were collated with common themes identified by one author (EK) and reviewed for agreement by a second author (LC).

\section{Ethical consideration}

Ethical approval was granted by the Ottawa Health Science Network Research Ethics Board prior to commencing the survey.

\section{RESULTS}

\section{EM department, division chairs, and chiefs}

Seventy department/division chairs and/or ED chiefs were invited to complete Survey 1, and 22 (31.4\%) completed it. Ten (45.5\%) worked in adult-only EDs, three (13.6\%) worked in pediatric-only EDs, and nine (40.9\%) worked in centres that see both adults and children.

\section{Formal training and skill capacity}

A majority of the respondents $(81.8 \%)$ reported at least one physician member on staff having formal training in QIPS, either in the form of a master's degree or certificate-level course. The focus of these was more often on quality improvement than on patient safety (Table 1). The majority of the respondents also had a formal leadership position for QIPS within their organization (82\%), with $83 \%$ of them having funding to support these positions. There was a variety of sources for this funding including hospital operational budgets (22\%), direct financial contributions from the EM physician group (22\%), provincial and government agency grants $(17 \%)$, university contributions $(6 \%)$, or a combination of these entities (Figure 1).

\section{Operational infrastructure}

Fifty percent of respondents reported having dedicated office space for their teams to conduct QIPS work, and 


\begin{tabular}{lc}
\hline Table 1. Number of survey respondents and percentage of \\
academic EDs with faculty members formally trained in QIPS \\
\hline Number of surveys sent & $\mathrm{N}$ \\
Department chair/chief (Survey 1) & 70 \\
Local QIPS lead (Survey 2) & 12 \\
Number of respondents & $\mathrm{n}(\%)$ \\
Department chair/chief & $22(31)$ \\
Local QIPS lead & $11(92)$ \\
Number of responding academic EDs with staff physicians & $\mathrm{n}(\%)$ \\
formally trained in quality improvement & \\
0 staff members & $4(18)$ \\
1 staff member & $5(23)$ \\
2 staff members & $6(27)$ \\
3 staff members & $3(14)$ \\
4 staff members & $1(5)$ \\
$5+$ staff members & $3(14)$ \\
Number of responding academic EDs with staff physicians & $\mathrm{n}(\%)$ \\
formally trained in patient safety & \\
0 staff members & $8(36)$ \\
1 staff member & $9(41)$ \\
2 staff members & $2(9)$ \\
3 staff members & $2(9)$ \\
4 staff members & $0(0)$ \\
5+ staff members & $1(5)$ \\
\hline ED = emergency department; QIPS = quality improvement and patient safety. & \\
\hline
\end{tabular}

$46 \%$ of them reported having provided administrative or support staff (defined as non-physician personnel dedicated to operational running of QIPS programs) to their teams. Almost all centres (91\%) have a dedicated quality committee, although only $65 \%$ are multidisciplinary; the rest comprised EM physicians only.

\section{Local QIPS leads}

From Survey 1, a total of 12 local QIPS leads were identified by their department/division chairs/chiefs and subsequently invited to participate in Survey 2. Of note, three of the authors (EK, LC, SM) also participated in the survey in their capacity as local QIPS leads.

\section{Educational activities}

Eleven (92\%) of them completed Survey 2. Further, $64 \%$ of respondents reported having a formal training program for teaching QIPS topics to EM residents. Seventy-one percent of these curricula had an option for the completion of an actual QI project. Three (27\%) centres had a QIPS education director position within their EM department to facilitate this QIPS training for residents, with two of them having formal funding for their positions. In contrast, only one (9\%) respondent reported having any QIPS topics addressing continuing professional development (CPD) activities for staff physicians, and none reported mandatory QIPS training as part of their annual review or reappointment process.

\section{Academic and scholarship}

Forty-five percent of the respondents reported that their department produced peer-reviewed QIPS publications over the previous five years, with a median of four (range: 1-10) manuscripts per centre. Over one-half of Survey 2 respondents (55\%) reported peer-reviewed QIPS abstract acceptances over the past five years, with a median of 10 (range: 1-100) abstracts per centre. Fifty-five percent of these QIPS projects had EM physicians as a project lead or co-lead. Over one-third (36\%) of respondents described formal funding for faculty members to carry out QIPS scholarship. Two (18\%) respondents reported that their department provides internal awards or has a points system for QIPS scholarship, and two (18\%) respondents reported that their group currently held external peer-reviewed QIPS grants. Access to research-specific infrastructure and supports for conducting QIPS scholarship were variable between the respondents' centres: mentorship (55\%), librarian (36\%), methodologist (27\%), administrative personnel (27\%), and statistician (9\%).

\section{QIPS activity}

Six respondents reported active ongoing QIPS projects at the time of the survey, ranging from 4 to 30 individual projects in progress per responding site. Physicians were the sole leads for these projects at one-third (2/6) of responding centres, and projects at the other two-thirds (4/6) of centres were co-led by physicians, nurses, allied health professionals, or any combination of these professionals. Only three respondents were aware of an explicit quality plan within their ED, of which all of them reported the direct linkage of QIPS projects to that quality plan.

\section{Qualitative comments}

In open-text comment boxes in both surveys, 17/22 (77\%) of ED chiefs and 5/11 (46\%) of local QIPS lead 


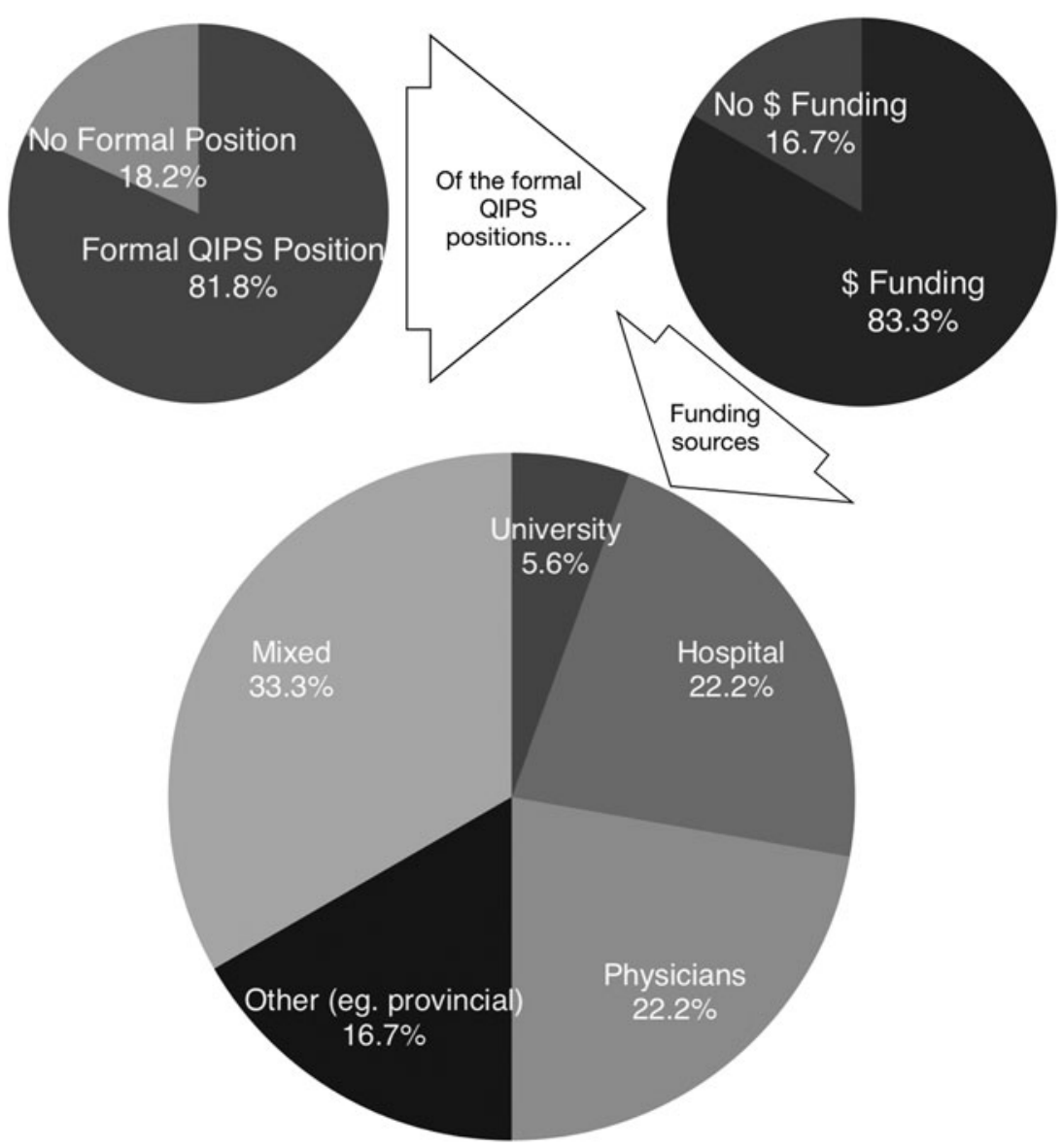

Figure 1. Proportion of academic EDs with formal OIPS positions and associated funding sources.

respondents described a number of perceived gaps and needs in relation to their QIPS activities. They identified, from a national perspective, a need for greater promotion and general awareness of the developing field of QIPS, guidance and consensus on high priority QIPS topics that all EDs can focus on, and a platform through which best practices and learnings from QIPS work can be shared and spread. At the local level, respondents identified a need for increased academic support to carry out QIPS work, greater support for the development of QIPS skills and capacity, and standardization of common QIPS indicators across EDs.

\section{DISCUSSION}

This study is the first environmental scan of QIPS activities in academic EM at Canadian medical schools and major teaching hospitals. We found that there is a significant level of engagement in QIPS, as evidenced by the large proportion of responding academic centres with dedicated positions for QIPS and recruitment of staff with QIPS training. There appears to be a keen interest in providing QIPS education in EM residency programs, with many centres providing longitudinal supervision for QI projects. We also found significant academic efforts related to QIPS activities, with a majority of responding centres successfully producing peerreviewed abstracts and manuscripts. In contrast, our study revealed that less than one-half of the responding centres have a dedicated administrative structure to support QIPS leads within their groups. Educational efforts are mostly focused on residents, with minimal investment in strengthening QIPS capacity for existing staff physicians. Despite the reported academic output, few respondents identified any formal funding or reward system to ensure ongoing academic success. Overall, our environmental scan found a disproportionate infrastructure in place to sustain QIPS activities in academic EM across Canada, and an opportunity for EDs to invest 
and close this potential gap in their efforts to advance QIPS in their departments further.

This study is important in summarizing current strengths and weaknesses of and providing an initial reference point for national and local QIPS activities in academic EM. Similar to other environmental scans for other academic endeavours, this report provides useful baseline information for comparison and highlights areas for improvement. ${ }^{21,22}$ On a national level, our results are guiding the CAEP QIPS committee's key strategic activities to develop recommendations and coordinate large-scale initiatives to support the growth of QIPS capacity across Canada. For example, our results revealed that a few centres were significantly more successful at producing a high volume of scholarly QIPS output; there is an opportunity for sharing successful strategies amongst all EM departments and perhaps leading to larger multicentre QIPS initiatives. Locally, individual centres can utilize these findings as a comparison to identify gaps in their QIPS efforts and set priorities for improvement, for example, developing and funding a formal QIPS position within their group, as is the case in the majority of responding centres. The results of this study highlight a significant imbalance between formal support and successful output of QIPS work in EM. This may form the basis for individual academic centres to advocate for increased funding to sustain this important work aimed at improving care for patients locally.

There were some limitations to this study. The generalizability of the findings is limited by the $31.4 \%$ response rate from chairs and chiefs. We intentionally expanded the initial invitation email list to include affiliated teaching sites, in addition to academic medical centres, to increase our reach, but this may have led to a decreased response rate. However, because smaller EDs might not have the same resources or support for QIPS activities within their EDs, we restricted our recruitment efforts to university-affiliated EDs. Our ethics board review did not allow us to link and identify individual responses; thus, we were unable to provide further demographic details about survey respondents. Another limitation of our study was the challenges associated with the identification of local QIPS leads within each organization (and whether they even had one), given the lack of an available list for these positions. We achieved an excellent response rate of 11 of the 12 participants for the local QIPS leads, but it remains unclear how many were simply not identified to our team by their respective chairs and chiefs.

This study represents a limited snapshot of QIPS activities in Canadian EM. Future research in this area will need to examine how such activities are supported, sustained, and broadened over time. One of the core tenants in the science of quality improvement is to measure the current state and continuously aim for improvements. A future comprehensive review of this field in Canadian EM should examine in more detail how QIPS work is evaluated and what impacts on clinical outcomes have been achieved. In addition, we will need to review whether national collaborations and spread of QIPS gains beyond local centres are ultimately successful.

\section{CONCLUSION}

This study is the first review of QIPS activities in EM across Canada. We found multiple EDs showing interest in and recognizing the importance of QIPS, with a demonstration of significant local educational and academic efforts. However, there appears to be a discrepancy between the level of formal support and infrastructure and such activities. These findings have informed the CAEP 2018 Academic Symposium QIPS panel recommendations. It will also continue to guide impactful national initiatives and provide local EDs with a starting point to advocate and advance their important QIPS efforts aimed at improving patient care.

\section{SUPPLEMENTARY MATERIAL}

The supplementary material for this article can be found at https://doi.org/10.1017/cem.2019.16

Acknowledgements: The authors would like to thank the CAEP office staff Shanna Scarrow, Kelly Wyatt, and Gisele Leger for their assistance with survey logistics, as well as Dr. Eddy Lang for enabling the formation of the QIPS panel for the 2018 Academic Symposium in Calgary.

Competing interests: None declared.

\section{REFERENCES}

1. Kohn LT, Corrigan JM, Donaldon MS, et al. To err is human: building a safer health system. Washington, DC: National Academy Press; 1999.

2. World Health Organization. Quality of care: patient safety: report by the secretariat. 2002. http://apps.who.int/gb/archive/pdf_files/WHA55/ea5513.pdf. 
3. Baker GR, Norton PG, Flintoft V, et al. The Canadian Adverse Events Study: the incidence of adverse events among hospital patients in Canada. CMA7 2004;170(11):1678-86.

4. James JT. A new, evidence-based estimate of patient harms associated with hospital care. 7 Patient Saf 2013;9(3):122-8.

5. Calder LA, Forster A, Nelson M, et al. Adverse events among patients registered in high-acuity areas of the emergency department: a prospective cohort study. C7EM 2010;12 (5):421-30.

6. Batalden PB, Davidoff F. What is "quality improvement" and how can it transform healthcare? Qual Saf Health Care 2007;16(1):2-3.

7. The Ethics of Improving Health Care Quality \& Safety: A Hastings Center/AHRQ Project, Mary Ann Baily, PhD, Associate for Ethics \& Health Policy, The Hastings Center, Garrison, New York, October, 2004

8. Health Council of Canada. Canada's quality improvement conundrum. Health Council of Canada; 2013. Available at: https://healthcouncilcanada.ca/files/HCC_Post-Symposium Program_EN.pdf.

9. Health Quality Ontario. Quality matters: Realizing excellent care for all. Health Quality Ontario; 2017. http://www.hqontario.ca/Portals/0/documents/health-quality/realizing-excellent-care-for-all-1704-en.pdf

10. Cheng AH, Barclay NG, Abu-Laban RB. Effect of a multidiagnosis observation unit on emergency department length of stay and inpatient admission rate at two Canadian hospitals. 7 Emerg Med 2016;51(6):739-747.e3.

11. Bandiera G, Gaunt K, Sinclair D, Trafford A. Emergency department overcrowding and long wait times: taking a corporate approach to improving patient flow. Healthc $Q$ 2014;17(4):34-40.

12. Soong C, High S, Morgan MW, Ovens H. A novel approach to improving emergency department consultant response times. BM7 Qual Saf 2013;22(4):299-305.

13. Kwok ES, Geymonat C, Peters K, et al. A novel emergency department surge protocol: implementation of a targeted response plan. 7 Clin Outcomes Manag 2015;22(11):495-503.
14. Liu C, Desai S, Krebs LD, et al. Effectiveness of interventions to decrease image ordering for low back pain presentations in the emergency department: a systematic review. Acad Emerg Med 2018;25(6):614-26.

15. Chartier LB, Josephson T, Bates K, Kuipers M. Improving emergency department flow through rapid medical evaluation unit. BMJ Qual Improv Rep 2015;4(1); epub, https:// doi.org/10.1136/bmjquality.u206156.w2663.

16. Chartier LB, Simoes L, Kuipers M, McGovern B. Improving emergency department flow through optimized bed utilization. BMF Qual Improv Rep 2016;5(1); epub, https://doi. org/10.1136/bmjquality.u206156.w2532.

17. Chartier LB, Cheng AH, Stang AS, Vaillancourt S. Quality improvement primer part 1: preparing for a quality improvement project in the emergency department. CFEM 2018;20 (1):104-11.

18. Chartier LB, Stang AS, Vaillancourt S, Cheng AH. Quality improvement primer part 2: executing a quality improvement project in the emergency department. CFEM 2018;20 (4):532-8.

19. Chartier LB, Vaillancourt S, Cheng AH, Stang AS. Quality improvement primer part 3: evaluating and sustaining a quality improvement project in the emergency department. CFEM 2018; epub, 1-8.

20. Chartier LB, Mondoux SE, Stang AS, Dukelow AM, Dowling SK, Kwok ES, Trivedi SV, Tepper J, Lang E. CAEP 2018 Academic Symposium: How do emergency departments and emergency leaders catalyze positive change through quality improvement collaborations? CFEM 2019; epub, 10.1017/cem.2019.25.

21. Stiell IG, Artz JD, Lang ES, et al. An environmental scan of academic emergency medicine at the 17 Canadian medical schools: why does this matter to emergency physicians? CFEM 2017;19(1):39-46.

22. Artz JD, Meckler G, Argintaru N, Lim R, Stiell IG. An environmental scan of academic pediatric emergency medicine at Canadian medical schools: identifying variability across Canada. CFEM 2018;20(5):693-701. 